\title{
Hypertension effects on p73 expression in the rat circumventricular organs and cerebrospinal fluid
}

\author{
Emilia M. Carmona-Calero, ${ }^{1,2}$, Ibrahim González-Marrero ${ }^{1,2}$, Manuela Castañeyra-Martin², \\ Juan M. González-Toledo ${ }^{1}$, Leandro Castañeyra-Ruiz ${ }^{2}$, Héctor de Paz-Carmona ${ }^{1}$, \\ Agustín Castañeyra-Ruiz ${ }^{2}$, Lidia Ruiz-Mayor ${ }^{1}$, Agustín Castañeyra-Perdomo ${ }^{1,2^{*}}$
}

${ }^{1}$ Departamento de Anatomía, Facultad de Medicina, Universidad de La Laguna, Tenerife, Islas Canarias, Spain

${ }^{2}$ Departamento de Biotecnología, Instituto de Investigación y Ciencias de Puerto del Rosario, Fuerteventura, Islas Canarias, Spain Email: ${ }^{*}$ acastane@ull.es

Received 14 December 2011; revised 30 January 2012; accepted 16 February 2012

\begin{abstract}
It has been reported that spontaneously hypertensive rats (SHR) show ventricular dilation, changes in CSF proteins and variations in the circumventricular organs (CVO) such as: the subcommissural organ (SCO), the subfornical organ (SFO) and the area postrema (AP) which are located in the walls of the third and fourth ventricles. On the other hand, p73 proteins are present in cells of the central nervous system (CNS) such as circumventricular structures and the neuroepithelium which are altered in ventricular dilation. The purpose of the present work is to study the TAp73 isoform expression in the circumventricular organs (CVO) and their variations in ventricular dilatation and arterial hypertension. Brains and cerebrospinal fluid (CSF) from control Wistar-Kyoto rats (WKY) and SHR were used. The paraffin sections containing the CVO were immunohistochemically processed with anti-TAp73 and by western blot, p73 bands in the CSF and circumventricular organ extract were also identified. The western blot study showed bands marked with p73 in the CSF and CVO, the p73 band expression was bigger in the SHR than in the WKY rats. We also found stronger markings in the SFO, SCO and AP of the hypertensive rats than in the WKY rats. It could be concluded that hypertension in the SHR produces alterations in the relationship between the p73 protein, circumventricular structures and CSF.
\end{abstract}

Keywords: Circumventricular Organs; p73; CSF; Hypertensive Rats

\section{INTRODUCTION}

Ritter and Dinh [1] described that spontaneously hypertensive rats present a progressive increase in ventricular

${ }^{*}$ Corresponding author. size from 4 to 56 weeks of age, and in some SHRs ventricle size increased to $270 \%$ of control and such ventricular dilation could be produced by a loss of grey and white matter similar to what occurs in hydrocephalus, for this reason, certain systemic and behavioural signs which are concomitant with hypertension in the SHR may be attributable to hydrocephalus and its neuropathological correlates [1]. It has also been observed in previous studies $[2,3]$ that the increase in ventricular size can be qualitatively observed after 15 weeks of postnatal age. On the other hand, alterations of the subcommissural organ, subfornical organ in the hypertensive and hydrocephalic rats were also observed [4-7].

The subcommissural organ ( $\mathrm{SCO})$ is a cerebral structure associated with the circulation and composition of the CSF, which secretes glycoprotein into the CSF where its greater part is condensed and forms Reissner's fibre (RF), and the other minor part remains soluble in the CSF. The human SCO has a great development during the foetal life $[8,9]$, variations in the CSF and SCO have been reported in different kinds of hydrocephalus [4,10, 11]. Alterations of the SCO in hydrocephalic mice and rats have been described, Takeuchi et al. [12] de- scribes agenesis of the SCO and the posterior commissure (PC) in hydrocephalic mice, Irigoin et al. [11], Rodriguez et al. [4] and Carmona-Calero et al. [7] found alterations in the secretion of the SCO in induced and spontaneously hydrocephalic rats. In addition, alterations in the secretions of cerebrospinal fluid proteins from the SCO have been described in spontaneously hypertensive rats [6].

The Subfornical Organ (SFO) is a circumventricular organ located below the commissure fornici, entering the rostral wall of the third ventricle [13], and is a neurogliovascular structure containing neurons, glia and plexus of fenestrated capillaries. The SFO is characterized by the absence of a blood-brain barrier [14]. The SFO has connections with the brain regions involved in the central regulation of drinking, salt appetite, blood pressure and 
cardiovascular function, such a region is the anteroventral region of the third ventricle which is involved in the control of drinking behaviour [15,16].

The area postrema located at end of the fourth ventricle under the obex [13] also contains neurons, glia and fenestrated capillaries, and does not have a blood-brain barrier. The AP functions are related with cardiovascular regulation and the catecholaminergic system as well as with the anteroventral region of the third ventricle $[17,18]$.

The role of p73 is significant in the central nervous system development, since the p73 knock-out mice present hippocampal dysgenesis, cortical hypoplasia and hydrocephalus, meaning that p 73 plays an important role in the different parameters regulating brain development [19]. The transactivating isoforms of p73 (TAp73) are similar to p53 acting as transcription factors inducing cellular apoptosis; on the other hand, the N-terminal truncated isoforms $(\triangle \mathrm{Np} 73)$ can inhibit the transcripttional function of p53 and TAp73 [20]. It has recently been reported that TAp73 is present in the SCO and CSF where it could play an important role in the maintenance of the ventricular wall and in the development of neuroepithelium proliferation [21].

Bearing in mind that SHRs present ventricular dilation, alterations in the circumventricular organs and CSF proteins variations, the purpose of the present work is to study the expression of p73 in the CSF, SCO, SFO and the AP in arterial hypertension and ventricular dilation.

\section{MATERIAL AND METHODS}

Brains and cerebrospinal fluid (CSF) from 16 control Wistar-Kyoto rats (WKY) and 16 spontaneously hypertensive rats (SHR) from Charles River Laboratories España S.A. (Barcelona, Spain) of 12 months of age were used. The rats were anesthetized with chloral hydrate $(200 \mu \mathrm{l} / 100 \mathrm{~g}$ of body weight at $160 \mathrm{mg} / \mathrm{ml})$ and $100 \mu \mathrm{l}$ of CSF from the cistern magna of each animal was extracted before sacrifice. Extracts of circumventricular organs and CSF were prepared from 12 rats from each group which were processed by protein electrophoresis according to Laemmli [22] (sodium docecyl sulfate-polyacrylamide gel electrophoresis SDS-PAGE, 5\% - 15\% gradient). Four rats from each group were fixed by intracardiac perfusion with Bruin's fluid, dehydrated and embedded in paraffin under standard conditions. Brains were cut into four serial coronal and sagittal sections of $10 \mu \mathrm{m}$ thickness. One of the serial coronal sections was stained by the Klüver-Barrera method.

The polyclonal antibody against TAp73 (Ab 14430, Abcam, Cambridge, UK) was used as the primary antibody. In each rat brain of each group, the sections at the level of the SCO, SFO and AP from the WKY and SHR were simultaneously incubated in the same coupling jar and each jar contained: anti-p73 1:1000. Incubation was for $24 \mathrm{~h}$ at room temperature, followed by "DAKO" StreptABCcomplex/HRP Duet, Mouse/Rabbit procedure. The peroxidase reaction product was visualized using diaminobenzidine reaction.

Western blots of CSF protein and SCO, SFO and AP extract protein were performed. The membranes with the blotted proteins were incubated in tris-saline (TBS) non-fat milk 5\% for 60 minutes and then incubated in the primary antibody anti-p73 1:1000 overnight. Anti-mouse IgG labelled with peroxidase (PIERCE) was used as the secondary antibody at a dilution of 1:80,000 for $1.45 \mathrm{~h}$ at room temperature. The peroxidase reaction products from western blot were visualized by quimioluminiscence (PIERCE). The primary antibody was omited to validate the control method specificity. The immunohistochemistry slides were converted to digital images by using an LEICA DMRB photomicroscope with an LEICA DC $300 \mathrm{~F}$ camera (Gemany). Image analysis was completed in Image $\mathrm{J}$ (v. $1.43 \mathrm{u}$, NIH, Bethesda, MD, USA). The "Mean Gray Value" was measured from the selected nuclei for all stained tissue and membranes. This value gives the average stain intensity in grayscale units for all threshold pixels. A single-factor analysis of variance (ANOVA) was used for the immunohistochemistry statistical study, which was conducted using the IBM SPSS statistic 19 software.

\section{RESULTS}

Hydrocephalus was clearly observed at 12 months of age in the SHRs by comparing the ventricular size of these SHRs with the normal ventricular size observed in WKYs of 12 months of age (Figures 2 (K), (L)).

\subsection{Immunohistochemistry}

The expression of the TAp73 in the SCO of WKY rats was present in apical perinuclear parts of ependymal and hypendymal cells and some marks were even observed in the ventricle (Figures 1(A), (C); 2(I), (J)). A significant increase of TAp73-ir $\left(\mathrm{F}_{1-6}=134.975 \mathrm{P}<0.05\right)$ was observed in the different parts of the ependymal and hypendymal cells in the SCO of the SHR (Figures 1(B), (E); 2(M), (N); 4).

The cytoplasm of cells in the ependyma layer was marked with TAp73-ir and this was also found in several body neurons and in perivascular spaces in the SFO of the WKY rats (Figures 1(A), (D); 2(A), (B)). A noteworthy increase of TAp73 expression $\left(\mathrm{F}_{1-6}=224.615 \mathrm{P}<\right.$ $0.05)$ in the parenchyma and neurons was found in the SFO hypertensive rats (Figures 1 (B), (F); 2(E), (F); 4).

The AP showed immunoreactive material in several neurons and perivascular spaces in the WKY rats (Figures 2(C), (D)) and a slight and not significant increase in the immunoreaction intensity was observed in neurons and ependyma in AP of the SHRs, (Figures 2(G), (H); 4). 

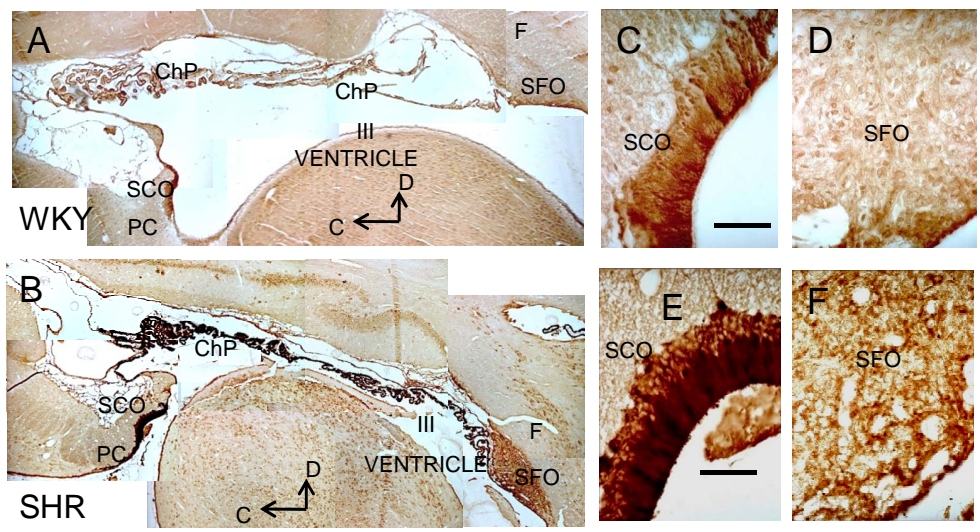

Figure 1. Sagittal section photographs of the rat brain immunostained with anti-TAp73. A: WKY rats; B: SHR rats; C: SCO, D: SFO of WKY rats; E: SCO; F: SFO of SHR rats. WKY: control Wistar-Kyoto rats; SHR: spontaneously hypertensive rats; SCO: subcommissural organ; SFO subfornical organ; $\mathrm{ChP}$ choroid plexus; F fornix; $\mathrm{PC}$ posterior commissure; III ventricle; $\mathrm{C}$ caudal; D dorsal. Bar $=80 \mu \mathrm{m}$.
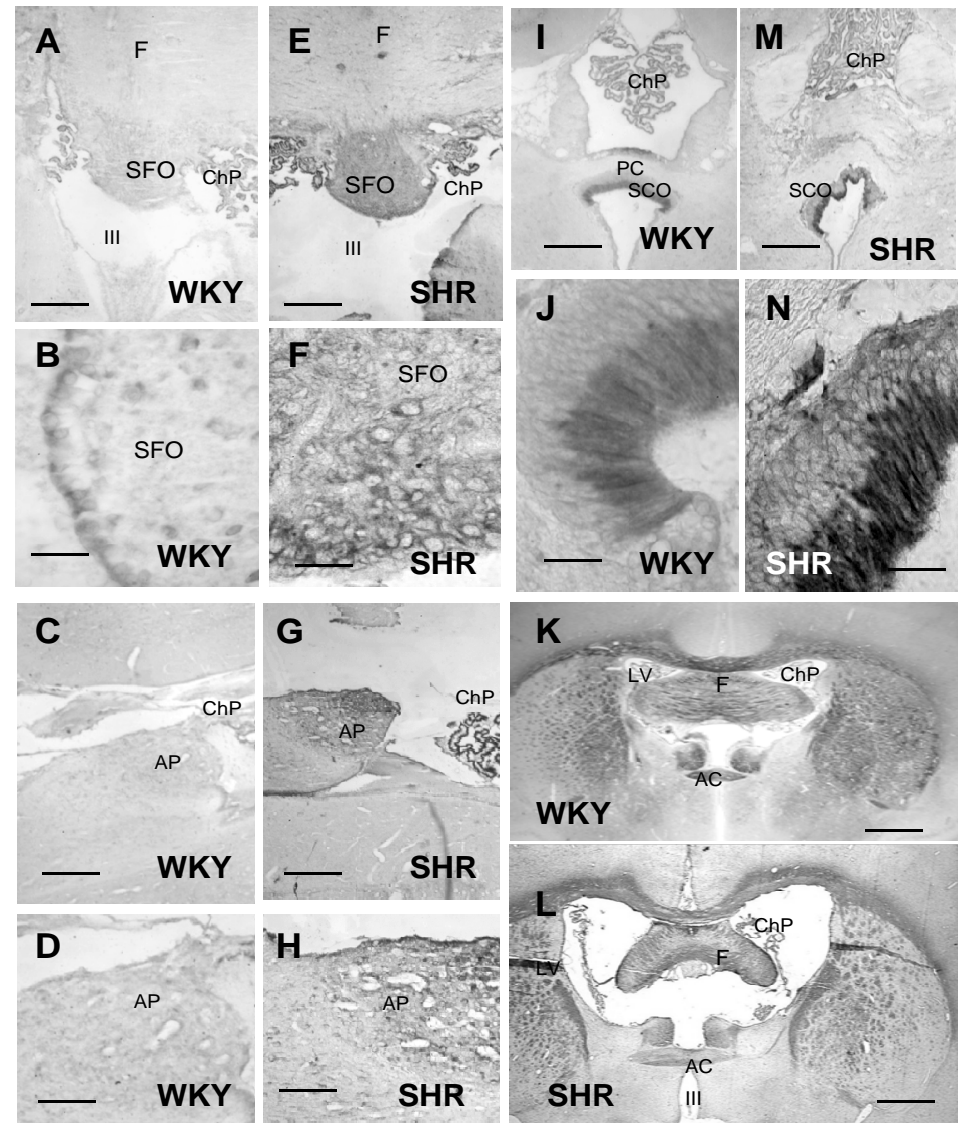

Figure 2. Photographs of the rat circumventricular organs immunostained with anti-TAp73. Coronal view of the SFO; A, B WKY rats; E, F SHR rats. Coronal view of the SCO; I, J WKY rats; M, N SHR rats. Sagittal view of the AP: C, D WKY rats, G,H SHR rats. Coranal view of the rat brain at the AC level: K, WKY rats, L SHR rats. Bar $=50 \mu \mathrm{m}(\mathrm{A}, \mathrm{E}, \mathrm{I}, \mathrm{M}, \mathrm{C}, \mathrm{G}) ; 150 \mu \mathrm{m}(\mathrm{B}, \mathrm{F}, \mathrm{J})$; $80 \mu \mathrm{m}(\mathrm{D}, \mathrm{H}) ; 300 \mu \mathrm{m}(\mathrm{K}, \mathrm{L})$. WKY: control Wistar-Kyoto rats; SHR: spontaneously hypertensive rats;AP: area postrema; SFO: subfornical organ; SCO subcommissural organ; OP optic chiasma; F fornix; III ventricle; $\mathrm{ChP}$ choroid plexus; LV lateral ventricle; AC anterior commissure. 


\subsection{Western Blot}

The anti-p73 reaction in the immunoblotting showed two bands of 65 and $90 \mathrm{kDa}$ in SCO, SFO and AP extracts which were more intense in the SCO $\left(\mathrm{F}_{1-10}=214.809 \mathrm{P}<\right.$ $0.05)$ of the SHRs WKY rats. There were also two bands of the same molecular weight in the cere- brospinal fluid, and the intensity of the reaction was greater in the CSF of the SHRs than in the WKY rats (Figures 3, 5).

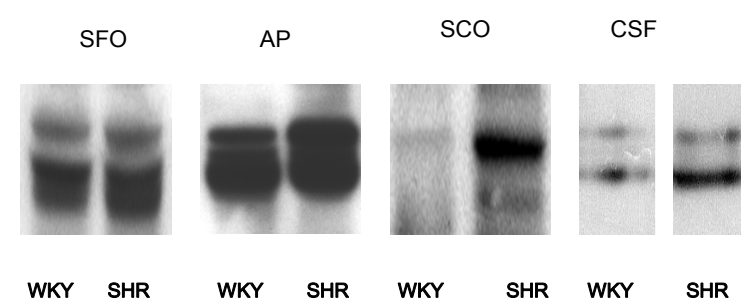

Figure 3. TAp73 western blot of the circumventricular organ extracts and cerebrospinal fluid. WKY: control Wistar-Kyoto rats; SHR: spontaneously hypertensive rats; AP: area postrema; SFO: subfornical organ; SCO subcommissural organ; CSF: cerebrospinal fluid.

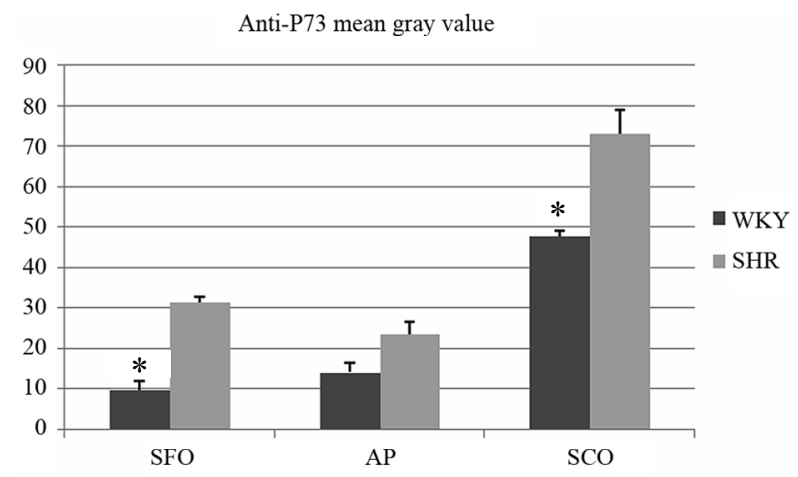

Figure 4. Densitometry of the circumventricular organs immunostained with anti-TAp73, *significant differences: SFO $\mathrm{F}_{1-6}=224.615 \mathrm{P}<0.05 ; \mathrm{SCO} \mathrm{F}_{1-6}=134.975 \mathrm{P}<0.05$.

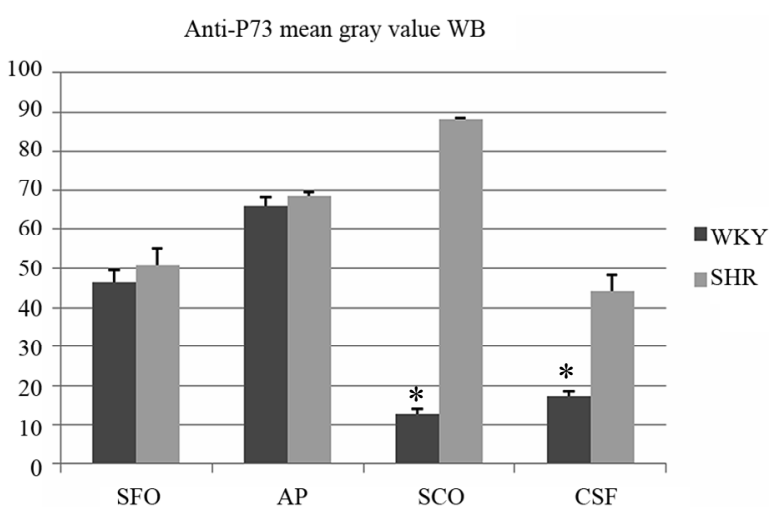

Figure 5. Densitometry of anti-TAp73 in the western blot of the circumventricular organ extract and CSF. " significant differences: SCO $\mathrm{F}_{1-10}=214.809 \mathrm{P}<0.05$; WKY: control Wistar-Kyoto rats; SHR: spontaneously hypertensive rats; AP: area postrema; SFO: subfornical organ; SCO subcommissural organ; CSF: cerebrospinal fluid.

\section{DISCUSSION}

The SHRs present an elevation of blood pressure from 10 weeks of age and a progressive increase of ventricular size from 4 to 56 weeks of age (Ritter and Dinh, 1986). Martinez-Peña y Valenzuela et al. [6] described that ventricular dilation can be qualitatively observed after 26 weeks of postnatal age, this is clearly present in 38 week old SHRs in the present work and a large ventricular dilation at 12 months of age was observed. On the other hand, ventricular enlargement occurred in the absence of p73, and consequently neuronal degeneration and tissue mass decreased, a phenomenon which is also observed in the degenerating human brain [21]. In addition, the balance between different isoforms of p73 is essential for the survival and cell death during brain development $[21,23]$. Pozniak et al. [20], indicating that $\mathrm{p} 73$ isoforms in the CNS are necessary for maintaining, at least, some populations of adult brain cells, therefore a balance in p73 isoforms is necessary for maintaining normal central nervous system development, a balance which could be altered in the SHR. The results of this study shows that chronic hypertension causes an increase in the reaction anti-p73 in the SFO of the SCO, and AP. Increased anti-p73 reaction in the CVO of the SHR compared to WKY rats was also observed after immunoblotting, which showed a p73-ir band in extracts of CVO, which was also stronger in the SHR. Moreover, p73 also identified bands in CSF, consistent with the conclusion that one of the isoforms of p73 is glycosylated and secreted into the cerebrospinal fluid [20]. These results could mean that hypertension and ventricular dilation induce alterations of the TAp73 in the CVO, in addition the TAp73 in the CSF may play an important role in the development of the ependymal layer and the neuroepithelium [24], which could explain the higher variations of TAp73 expression found in the subcommissural organ (SCO) and in the CSF.

Recent study showed that mice lacking the TAp73 isoform, have alterations in dentate gyrus of the hippocampus [25], an important neurogenic structure in the adult brain. These results, and the relations among the p53 family, stem cells, and aging, suggest that TAp73 may play a role not foreseen in maintenance of neural stem cells. This hypothesis has been tested and shows that TAp73 ensures normal adult neurogenesis by promoting the longterm maintenance of neural stem cells [26].

High blood pressure in SHRs is accompanied by ventriculomegaly and variations of TAp73 expression in circumventricular organs and the CSF, therefore, if hypertension aggravates the aging brain and impairs memory, attention and abstract reasoning [27-29], and that the TAp73 in the CSF plays an important role in maintaining the adult ependyma, ventricular wall, and neural stem 
cells, one might conclude that TAp73 appears to play a role in maintaining the ventricular system and circumventricular structures, and that alterations of TAp73 CSF levels in hypertension may be an important factor that should be taken into account in the development of premature aging and cognitive disorders.

\section{ACKNOWLEDGEMENTS}

This work was supported by the Fundación Canaria de Instituto de Investigacion y Ciencias de Puerto del Rosario (INIPRO) project n 01/08.

\section{REFERENCES}

[1] Ritter, S. and Dinh, T.T. (1986) Progressive postnatal dilation of brain ventricles in spontaneously hypertensive rats. Brain Research, 370, 327-332. doi:10.1016/0006-8993(86)90488-9

[2] Carmona-Calero, E., Pérez-González, H., Martínez-Peña y Valenzuela, I., González-Marrero, I., Pérez-García, C.G., Marrero-Gordillo, N., Ormazabal-Ramos, C., Castañeyra-Perdomo, A. and Ferres-Torres, R. (2005) Effect of the arterial hypertension and captopril treatment on the angiotensin II content in the subfornical organ. A study in SHR rats. Histology and Histopathology, 20, 135-138.

[3] González-Marrero, I., Carmona-Calero, E.M., FernándezRodríguez, P., Pérez-González, H., Ormazabal-Ramos, C., Castañeyra-Ruiz, L., Pérez-García, C.G., Martínez-PeñaValenzuela, I., Castañeyra-Ruiz, A., Castañeyra-Perdomo, A. and Ferres-Torres, R. (2007) Expression of certain proteins in the subfornical organ and cerebrospinal fluid of spontaneously hypertensive rats. Histology and Histopathology, 22, 1371-1378.

[4] Rodríguez E.M., Oksche, A., Hein, S. and Yulis, C.R. (1992) Cell biology of the subcommissural organ. International Review of Cytology, 2, 39-121.

[5] Castañeyra-Perdomo, A., Carmona-Calero, E., Meyer, G., Perez-Gonzalez, H., Pérez-Delgado, M.M., MarreroGordillo, N., Rodríguez, S. and Rodríguez, E.M. (1998) Changes in the secretory activity of the subcommissural organ of spontaneously hypertensive rats. Neuroscience Letters, 246,133-136.

doi:10.1016/S0304-3940(98)00252-3

[6] Martínez-Peña y Valenzuela, I., Carmona-Calero, E.M., Pérez-González, H., Ormazabal-Ramos, C., FernándezRodríguez, P., González-Marrero, I., Castañeyra-Perdomo, A. and Ferres-Torrer, R. (2006) Alterations of the cerebrospinal fluid proteins and subcommissural organ secretion in the arterial hypertension and ventricular dilatation. A study in SHR rats. Histology and Histopathology, 21, 179-185.

[7] Carmona-Calero, E.M., González-Marrero, I., GonzálezToledo, J.M., Castañeyra-Ruiz, A., De Paz-Carmona, H., Castañeyra-Ruiz, L., Fernández-Rodríguez, P., RuizMayor, M.L. and Castañeyra-Perdomo, A. (2009) Reissner's fibre proteins and p 73 variations in the cerebrospinal fluid and subcommissural organ of hydrocephalic rats. Anatomia, Histologia, Embryologia, 38, 282-285. doi:10.1111/j.1439-0264.2009.00939.x

[8] Castañeyra-Perdomo, A., Meyer, G. and Ferres-Torres,R. (1985) The early development of the human subcommissural organ. Journal of Anatomy, 143, 195-200.

[9] Castañeyra-Perdomo, A., Carmona-Calero, E.M, PérezGonzáles, H., Martínez-Peña y Valenzuela, I., PlazaMoreno, P., Ormazabal-Ramos. C. and GonzálezMarrero, I., Trujillano-Dorado, A. and Ferres-Torres, R. (2004) Ontogenic development of the human subcommissural organ. European Journal of Anatomy, 8, 107120.

[10] Castañeyra-Perdomo, A., Meyer, G.,. Carmona-Calero, E, Bañuelos-Pineda, J., Méndez-Medina, R., OrmazabalRamos, C. and Ferres-Torres, R. (1994) Alterations of the subcommissural organ in the hydrocephalic human fetal brain. Developmental Brain Research, 79, 316-320. doi:10.1016/0165-3806(94)90138-4

[11] Irigoin, C., Rodriguez, E.M., Heinrichs, M., Frese, K., Herzog, S., Oksche, A. and Rott, R. (1990) Immunocytochemical study of the subcommissural organ of rats with induced postnatal hydrocephalus. Experimental Brain Research, 82, 384-392.

doi:10.1007/BF00231257

[12] Takeuchi, I.K., Kimura, R., Matsuda, M. and Shoji, R. (1987) Absence of subcommissural organ in the cerebral aqueduct of congenital hydrocephalus spontaneously occurring in MT/HokIdr mice. Acta Neuropathologica, 73, 320-322. doi:10.1007/BF00688253

[13] Castañeyra-Perdomo, A., Meyer, G. and Heylings, D.J. (1992) Early development of the human area postrema and subfornical organ. The Anatomical Record, 232, 612 619. doi:10.1002/ar.1092320416

[14] Akert, K. and Steiner, F.A. (1970) The ganglion psalterii. A brief review of anatomical and physiological aspects of the subfornical organ in mammals. Bibliotheca Psychiatrica, 10, 1-14.

[15] Lenkei, Z., Corvol, P. and Llorenz-Cortes, C. (1995) The angiotensin receptor subtype AT1A predominates in rat forebrain areas involved in blood pressure, body fluid homeostasis and neuroendocrine control. Molecular Brain Research, 30, 53-60. doi:10.1016/0169-328X(94)00272-G

[16] Ushigome, A., Nomura, M. and Tanaka, J. (2004). Modulation of noradrenaline release in the median preoptic area by GABAergic inputs from the organum vasculosum of the lamina terminalis in the rat. Neurochemistry International, 44, 139-144. doi:10.1016/S0197-0186(03)00134-7

[17] Brody, M.J. (1988) Central nervous system and mechanisms of hypertension. Clinical Physiology and Biochemistry, 6, 230-239.

[18] Saper, C.B., Reis, D. and Joh, T. (1983) Medullary catecholamine inputs to the anteroventral third ventricular cardiovascular regulatory region in the rat. Neuroscience Letters, 42, 285-291. doi:10.1016/0304-3940(83)90276-8

[19] Meyer, G., Cabrera-Socorro, A., Perez-Garcia, C.G., Martinez-Millan, L., Walker, N. and Caput, D. (2004) Developmental roles of p73 in Cajal-Retzius cells and 
cortical patterning. Neuroscience, 24, 9878-9887. doi:10.1523/JNEUROSCI.3060-04.2004

[20] Pozniak, C.D., Radinovic, S., Yang, A., McKeon, F., Kaplan, D.R. and Miller, F.D. (2000) An anti-apoptotic role for the p53 family member p73, during developmental neuron death. Science, 289, 304-306. doi:10.1126/science.289.5477.304

[21] Cabrera-Socorro, A., Pueyo Morlans, M., Suarez Sola, M., González Delgado, F.J., Castañeyra Perdomo, A., Marín, M.C. and Meyer, G. (2006) Multiple isoforms of the tumor protein $\mathrm{p} 73$ are expresssed in the adult human telencephalon and choroid plexus and present in the cerebrospinal fluid. European Journal of Neuroscience, 23, 2109-2118. doi:10.1111/j.1460-9568.2006.04750.x

[22] Laemmli, U.K., (1970) Cleavage of structural proteins during the assembly of the head of bacteriophage T4. Nature, 227, 680-685. doi:10.1038/227680a0

[23] Wilson, C., Henry, S., Smith, M.A. and Bowser, R. (2004) The p53 homologue p73 accumulates in the nucleus and localizes to neurites and neurofibrillary tangles in Alzheimer disease brain. Neuropathology and Applied Neurobiology, 30, 19-29. doi:10.1046/j.0305-1846.2003.00496.x

[24] Cabrera-Socorro, A., Hernandez-Acosta, N.C., GonzálezGomez, M. and Meyer, G. (2007) Comparative aspects of p73 and Reelin expression in Cajal-Retzius cells and the cortical hem in lizard, mouse and human. Brain Research,
1132, 59-70. doi:10.1016/j.brainres.2006.11.015

[25] Tomasini, R., Tsuchihara, K., Wilhelm, M., Fujitani, M., Rufini, A., Cheung, C.C., Khan, F., Itie-Youten, A., Wakeham, A., Tsao, M.S., Iovanna, J.L., Squire, J., Jurisica, I., Kaplan, D., Melino, G., Jurisicova, A. and Mak, T.W. (2008) TAp73 knockout shows genomic instability with infertility and tumor suppressor functions. Genes \& Development, 22, 2677-2691. doi: $10.1101 / \mathrm{gad} .1695308$

[26] Fujitani, M., Cancino, G.I., Dugani, C.B., Weaver, I.C., Gauthier-Fisher, A., Paquin, A., Mak, T.W., Wojtowicz, M.J., Miller, F.D. and Kaplan, D.R. (2010) TAp73 acts via the bHLH Hey2 to promote long-term maintenance of neural precursors. Current Biology, 20, 2058-2065. doi:10.1016/j.cub.2010.10.029

[27] Jennings, J.R. and Zanstra, Y. (2009) Is the brain the essential in hypertension? NeuroImage, 47, 914-921. doi:10.1016/j.neuroimage.2009.04.072

[28] Raz, N. and Rodrigue, K.M. (2006) Differential aging of the brain: Patterns, cognitive correlates and modifiers. Neuroscience \& Biobehavioral Reviews, 30, 730-748. doi:10.1016/j.neubiorev.2006.07.001

[29] Waldstein, S.R., Giggey, P.P., Thayer, J.F. and Zonderman, A.B. (2005) Nonlinear relations of blood pressure to cognitive function: The Baltimore longitudinal study of aging. Hypertension, 45, 374-379. doi:10.1161/01.HYP.0000156744.44218.74 